\title{
WebDL: Un sistema adaptativo de educación a distancia a
} través de Internet

\author{
(WebDL: An Adaptive Web-Based Educational System for Distance Learning)
}

\author{
JESÚS GONZÁLEZ BOTICARIO \\ ELENA GAunIoso VÁZQUEZ \\ FÉLIX HERNÁNDEZ DEL OLMO
}

\author{
Departamento de Inteligencia Artificial \\ Universidad Nacional de Educación a Distancia
}

(España)

\begin{abstract}
RESUMEN: La enseñanza universitaria a distancia se está beneficiando del uso intensivo de los recursos ofrecidos por Internet. La variedad de información y servicios ofertados unidos a la naturaleza dispar del alumnado dificultan el aprovechamiento de este medio. Para paliar estos problemas y otros relacionados se está desarrollando un sitio web en el que se facilita un acceso personalizado a dichos recursos. Este sitio se apoya en tres elementos fundamentales: nuevos materiales docentes para aprovechar las venta fas del medio, una organización docente alternativa adaptada a las necesidades de los distintos protagonistas (alumnos, profesores y tutores) y un sistema interactivo, WebDL, que facilita un acceso personalizado y adaptativo a las necesidades de información y de comunicación de cada usuario en cada instante. Hasta el momento se están desarrollando los recursos y el material relacionado con tres asignaturas de los estudios de informática de la UNED, cuya docencia depende del Departamento de Inteligencia Artificial. Este artículo se centra en la descripción del sistema WebDL, sus componentes y su funcionalidad
\end{abstract}

\section{Sistema educativo adaptativo basado en la web - Aprendizaje a distancia - Hipes-media adaptativo - Sistemas inteligentes de tutoría - Inteligencia artificial}

ABSTRACT: University distance learning is benefiting from the intensive use of Internet resources. The variety of information and services offered together with different kinds of students are impeding efficient use of this nzedium. In order to alleviate these problems and other associated ones, a web Site lS being developed to facilita te personalized access to these resources. This site is based on three essential elements: new teaching materials to make the most of the medium, alterno tive teaching organization adapted to the requirements of the different protagonists (students, lecturers and tutors) and an interactive system, WebDL, which facilitates personalized and adaptive access to information and communication requirements to each and every user at each and every moment. To date, the resources and material for three computer studies courses are being developed at UNED, whose de-livery depends on the Artificial Intelligence Department. This paper focuses in WebDL's description, its componentS and its functionality.

\section{Web-Based Adaptive Educational System - Distance-Learning - Adaptive Hypermedia - Intelligent Tutoring Systems - Artificial Intelligence.}

\section{INTRODUCCIÓN}

La educación a través de Internet es un campo de investigación y de desarrollo que está acaparando cada vez más atención. Las ventajas de la enseñanza basada en la web son claras: independencia del lugar de estudio, permite el acceso de gran número de alumnos, permite compaginar el estudio con otras actividades, etc. Un curso basado en la web únicamente debe ser instalado y mantenido en un sólo 
servidor mientras que puede ser usado por un número ilimitado de alumnos. Como fruto de los trabajos realizados tanto en el campo de la informática como en el de educación en los últimos años han aparecido gran número de cursos que se imparten a través de Internet, así como otras aplicaciones educativas (sistemas de autoevaluación, clases programadas, etc.).

De hecho, es indudable que el buen uso de los medios actualmente disponibles en Internet está suponiendo ya, un cambio radical en las relaciones de enseñanzaiaprendizaje. El profesor se está convirtiendo en un facilitador de información, analista crítico de áreas de conocimiento, guía de estudio, revisor y evaluador de la capacitación del alumno. El alumno debería, por tanto, empezar a ser consciente de su papel esencialmente activo en el proceso de aprendizaje, como miembro de una comunidad virtual de personas con unos intereses de formación compartidos.

Relacionadas con esta perspectiva, más allá del marco de la educación a distancia, existen cada vez más iniciativas en la Web basadas en el desarrollo de comunidades virtuales de intereses diversos, comunidades de personas con niveles de experiencia diversos y con intereses comunes que se unen mediante Internet para beneficiarse de la comunicación, colaboración y coordinación (Boticario y Gaudioso, 2000a).

Frente a estas prometedoras experiencias se detecta en numerosos centros educativos on-line la creciente dificultad en atender las demandas tan variadas de los distintos tipos de usuarios, además de la falta de estructuración y la dispersión de las fuentes ofertadas en la Web (foros de debate, listas de distribución, páginas de diverso tipo: institucionales, asignaturas, preguntas más frecuentes, profesores, prácticas, pruebas de evaluación a distancia...), por lo que se dificulta notablemente el acceso a la información o al servicio deseado. Por otro lado, se comprueba que el aprovechamiento de los recursos ya disponibles depende mucho del grado de formación del usuario en su manejo y del conocimiento de la propia estructura del web site. Además, silo que se pretende es impartir un curso a través de este medio se le debe proporcionar al alumno un apoyo similar al que recebiría en la enseñanza presencial por parte de sus compañeros y prof esores, y no limitarse a construir un conjunto de páginas HTML estáticas con los contenidos del curso.

Con el propósito de resolver estos problemas y otros relacionados en el marco de las necesidades que caracterizan a la Universidad Nacional de Educación a Distancia, estamos desarrollando un sitio web que se apoya en tres elementos fundamentales. En primer lugar, se proporcionan servicios docentes concretos de información y de comunicación para satisfacer las necesidades de los distintos alumnos y profesores (Boticario, 1997 a). En segundo término, se ofertan nuevos tipos de material (Boticario, 1 997b) que intentan explotar las posibilidades del medio. Finalmente, se facilita un acceso personalizado a los recursos y servicios disponibles basado en el uso de un sistema aprendiz interactivo capaz de adaptarse a las necesidades de información y de comunicación de cada usuario en cada instante (Boticario y Gaudioso, 1999, 2000b, 2000c).

Este trabajo se centra en la descripción del sistema, WebDL, que nos permite proporcionar al alumno un acceso personalizado a los contenidos del web-site educativo. En las secciones siguientes describiremos los fundamentos del sistema y la arquitectura del mismo; por último describiremos una interacción típica con un usuario.

La aplicación del sistema que aquí se resalta consiste en la personalización de las prácticas de las asignaturas de aprendizaje de la Escuela Universitaria de Informática (EUI) y de los cursos de tercer ciclo del Departamento de Inteligencia Artificial de la UNED. Antes de abordar dicha experimentación y de presentar los fundamentos en los que se apoya conviene precisar que este sistema se ha realizado partiendo de un estudio exhaustivo de las fuentes disponibles que comprende: las experiencias de otros centros de enseñanza a distancia, las tendencias de los entornos interactivos de aprendizaje (McArthur et al., 1993) y el software disonible más apropiado (Boticario y Gaudioso, 1999).

\section{FUNDAMENTOS}

El desarrollo de esta aplicación se apoya en tres elementos destacados: en el cumplimiento efectivo de unas normas de diseño que garantice un acceso eficiente a la información disponible, en la 
personalización de la interacción como vía de enriquecimiento y agilización de dicho acceso y en una organización conceptual estructurada y flexible de los contenidos.

Puesto que nuestro objetivo es permitir el acceso al sistema con unos requerimientos mínimos, la interacción del alumno con el servidor se realizará a través de un navegador convencional sin necesidad de software específico adicional. Por tanto, un elemento fundamental a considerar será el diseño de las páginas HTML que compondrán el material que se presenta al alumno: así se prima la efectividad y los contenidos frente a la espectacularidad y el derroche de medios; para ello nos apoyamos en las recomendaciones de Jackob Nielsen ${ }^{1}$.

1 www.useit.com/a1ertbox.

Considerando las ventajas del aprendizaje colaborativo, la multitud de tareas independientes involucradas, la impredecibilidad del resultado y la dispersión de los recursos existentes se ha optado por una arquitectura multiagente; más concretamente, por un Sistema de Decision Multiagente (MADS) (Giraldez y Borrajo, 1998) capaz de coordinar las respuestas dadas por diferentes agentes con capacidades de aprendizaje distintas adaptadas a cada tipo de problema relacionado con la única respuesta global (de ahí que se considere sistema de decisión) que se quiere dar en cada instante. La comunicación entre agentes se realiza utilizando el lenguaje KQML (Finin et al., 1994) y el contenido de los mensajes se representa mediante KIF (Genesereth y Fikes, 1992). Los conceptos intercambiados en los mensajes dependen de las ontologías utilizadas, que en este caso son específicas de sistemas de educación (Chen y Mizoguchi, 1999).

En definitiva, el sistema es el resultado de una combinación efectiva de técnicas aplicadas en sistemas de educación inteligentes (ITS) (Weber y Specht, 1997; Nakabayashi, 1996), programas hipermedia adaptativos (AH) (Brusilovsky, 1996; Brusilovsky et al., 1996) y sistemas aprendices2 (Dent et al., 1992) . Se en- marca, por tanto, dentro de los llamados sistemas de educación adaptativos en la Web (Brusilovsky, 1998). Con el enfoque adoptado de sistemas aprendices se pretende expandir la base de conocimiento inicial a la vez que se busca reducir el esfuerzo del usuario en la toma de decisiones (hipermedia adaptativo).

Finalmente, con respecto a la organización conceptual de los contenidos, las prácticas así como los pasos aconsejados de resolución de los ejercicios se basan en el modelo propuesto por Roger Schank en el sistema ASK (Schank y Cleary, 1995), donde los contenidos se presentan mediante una red de conceptos (nodos) prefijados por el tutor que deben ser aprendidos por el alumno y los arcos representan la transición de un concepto a otro en función de los conocimientos que el alumno va aprendiendo y los intereses del propio alumno. Dichas transiciones se presentan en forma de preguntas o de opciones que el alumno va eligiendo.

\section{DESCRIPCIÓN DEL SISTEMA}

El sistema está implementado como un sistema de decisión multiagente organizado como sigue. Los dos componentes principales son: la interacción con el usuario y el módulo adaptativo. El primero se realiza a través del agente de interfaz y se encarga de la presentación organizada de los diferentes tipos de material conforme a unas normas de diseño que buscan la máxima usabilidad. El módulo adaptativo está formado por los siguientes agentes: agente de modelo, agente de modelado, agente de material, agente pedagógico, agente de contacto, agente de identificación de servicios, agente del servicio, agente de modelado del servicio y agente coordinador. Los cuatro primeros proporcionan la funcionalidad básica de un ITS. Los cuatro siguientes sirven para identificar los servicios del sistema que han resultado de interés para un conjunto de usuarios mediante filtrado colaborativo. Finalmente, el agente coordinador es el encargado, por un lado, de distribuir las peticiones a los agentes relacionados con la petición del usuario y, por otro, de confeccionar una única respuesta para el agente interfaz (ver Figura 1).

2 Asistentes personalizados que aprenden a partir de la observación de las acciones del usuario en la gestión de los elementos de un dominio específico. 


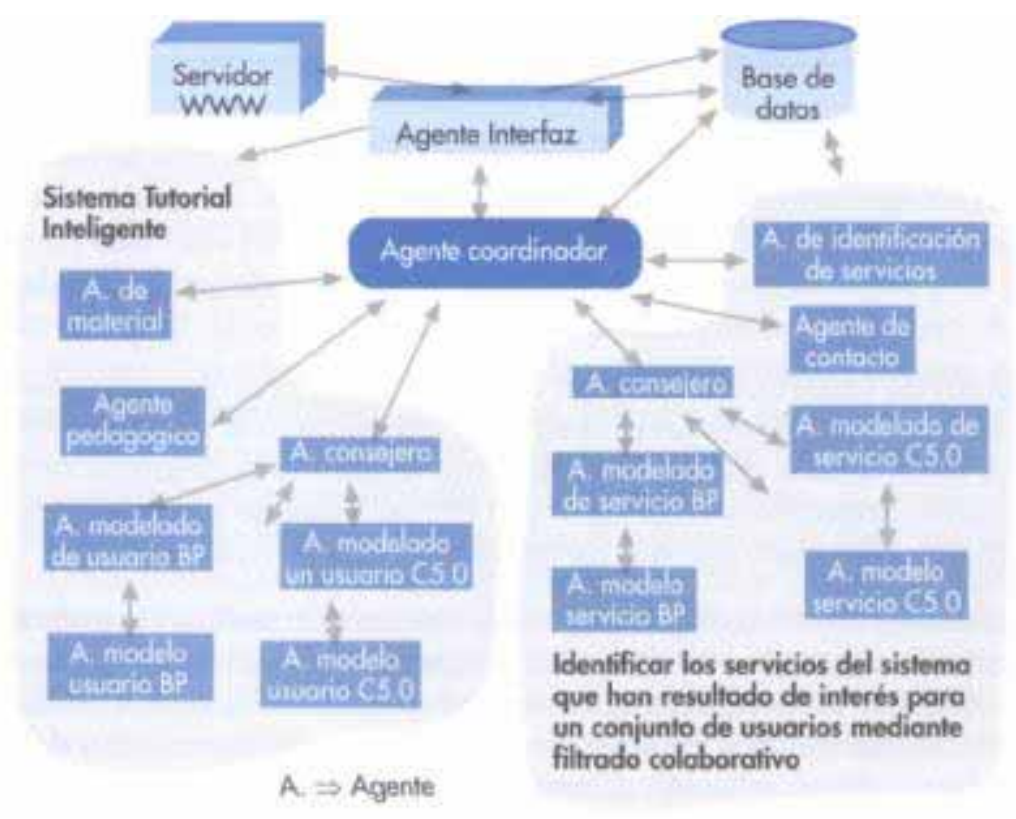

Figura 1. Arquitectura general del sistema WebDL

De esta forma cada vez que un alumno realiza una petición cada agente proporciona una respuesta en función de la tarea de la que está encargado (todos los agentes trabajan en paralelo con lo que la respuesta del sistema es más eficiente (Boticario y Gaudioso, 2000b)).

Cada agente dispone de una base de conocimiento propia, donde se definen las entidades que maneja, relaciones entre ellas, ...; los agentes que realizan tareas de aprendizaje son capaces de ampliar su base de conocimiento de forma dinámica, añadiendo nuevas entidades y modificando las ya existentes, según el alumno vaya interactuando con el sistema (se ajustan a lo que se ha denominado como sistemas aprendices). Las bases de conocimiento de los agentes se encuentran representadas utilizando THEO (Mitchell et al., 1990), un entorno genérico de programación orientado al desarrollo de sistemas capaces de olucionar problemas con capacidad autónoma de mejorar su comportamiento. Los elementos básicos de la arquitectura proporcionados en THEO comprenden mecanismos de inferencia, aprendizaje y representación del conocimiento.

Para acelerar la respuesta del sistema se vuelca en una base de datos el modelo que mejor valoración haya recibido hasta el momento, siempre y cuando el porcentaje de aciertos de dicho modelo supere un $90 \%$. Con este planteamiento se pretenden acumular en la base de datos todos los modelos (de usuario y de servicio) que el sistema ha podido comprobar que están totalmente aprendidos.

\section{INTERACCIÓN CON EL USUARIO}

El sistema WebDL no sólo pretende guiar al alumno a través de los contenidos del curso (aconsejándole enlaces, generando ejercicios de autoevaluación, etc.), sino que le permite utilizar todos los servicios que ofrece el sitio web (foros, chats, espacios de trabajo compartido, etc.) de una forma cómoda y sencilla adaptándose al nivel de pericia que el alumno ha demostrado en el uso de dichos servicios.

Cuando un usuario interacciona con el sistema, una vez se ha identificado, se le presentan en páginas web dinámicas los elementos del dominio (cursos, ejercicios relacionados con los ya realizados, temario y asignaturas de interés...) y los servicios disponibles en el sitio web (foros, listas de correo, anotaciones...) que el sistema considera que son de interés para dicho usuario.

El usuario percibe el dinamismo en la interacción mediante: presentaciones de información diferente (resaltar, ocultar, realizar anotaciones), alteraciones del orden en que aparecen los elementos del interfaz, cambios en el nivel de interacción (abriendo nuevas posiblidades de acuerdo a la pericia inferida del usuario) y descripciones de información diferente. 
La interfaz del usuario consta de dos áreas de trabajo claramente diferenciadas (ver Figura 2) cuando los consejos del sistema no puedan ser integrados en la información presentada (por ejemplo, en la parte superior se accede a un foro de discusión y en la parte inferior se muestran otros foros y listas de distribución relacionados) o cuando la información aconsejada sea contextual y no afecte directamente a la información presentada (por ejemplo, cuando en la parte superior se muestra el temario de una asignatura $y$ en su parte inferior se ofrecen asignaturas con temarios relacionados).

Las tareas de personalización que realiza en este momento el sistema son las de soporte adaptativo a la navegación y la de soporte adaptativo a la colaboración (Brusilovsky, 1996; Boticario et. al, 2000).

Vamos a explicar, a continuación, en qué consisten dichas tareas de personalización. La tarea de soporte adaptativo a la navegación consiste en guiar al alumno a través de los contenidos de las páginas del servidor web mediante las anotaciones que se hacen en los enlaces recomendados por el sistema.

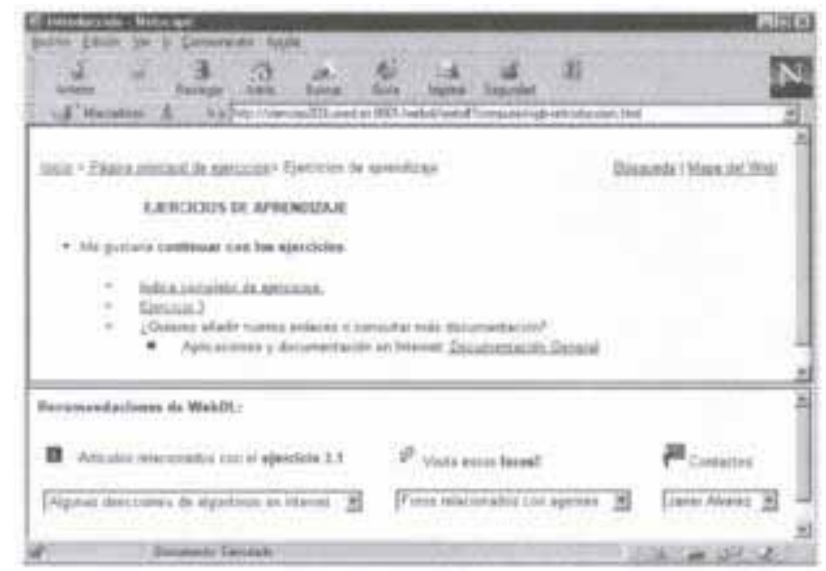

Figura 2. Dos áreas de trabajo en el interfaz de WebDL

En la Figura 3 se muestra el proceso de inicialización del sistema para que pueda impartir un curso a través de Internet. Además de poder incorporar al curso páginas HTML que ya están construidas, el diseñador del curso tiene la posibilidad de diseñar el material especifi'camente para el curso. El profesor deberá contar con una serie de ficheros que guardan el contenido de cada una de las páginas del curso; con estos ficheros, el profesor construye el material que se impartirá en el curso, definiendo las posibles reglas de tutorización (por ejemplo, "si el alumno ha visitado una cierta página entonces será conveniente aconsejarla otra página en concreto”), temas de los que trata la página, etc.... Por el momento, el profesor debe especificar todos estos datos directamente utilizando THEO, pero está previsto implementar un diseñador que permita realizar esto sin necesidad de que el profesor utilice THEO.

Una vez que se han definido los contenidos que se quieren impartir en el sistema, el alumno puede empezar a utilizar el sistema. Tal y como se muestra en la Figura 3, es aconsejable proporcionar a WebDL los datos personales y el historial académico del alumno, de forma que el sistema disponga de más datos que le permitirán realizar un mejor modelado del alumno.

En la Figura 4 se muestra la pantalla de entrada al sistema. En ella se le pide al alumno la identificacion de usuario y su contraseña, para poder identificarlo con su modelo de usuario correspondiente. Si es la primera vez que el alumno se conecta con el sistema, éste inicializa automáticamente un modelo de usuario con una serie de datos por defecto (el nivel de interés en el curso es medio, el medio de contacto es el servidor web, etc.) que se irán modificando según vaya interactuando el alumno con el servidor.

Cuando el alumno se conecta con WebDL, se encuentra con una página de entrada generada dinámicamente de acuerdo a su modelo de usuario y a las interacciones hechas en sesiones anteriores; si es la primera vez que se conecta, dicha página de entrada se construye de acuerdo a los valores por defecto que se asignan al modelo cuando se da de alta a un usuario. 


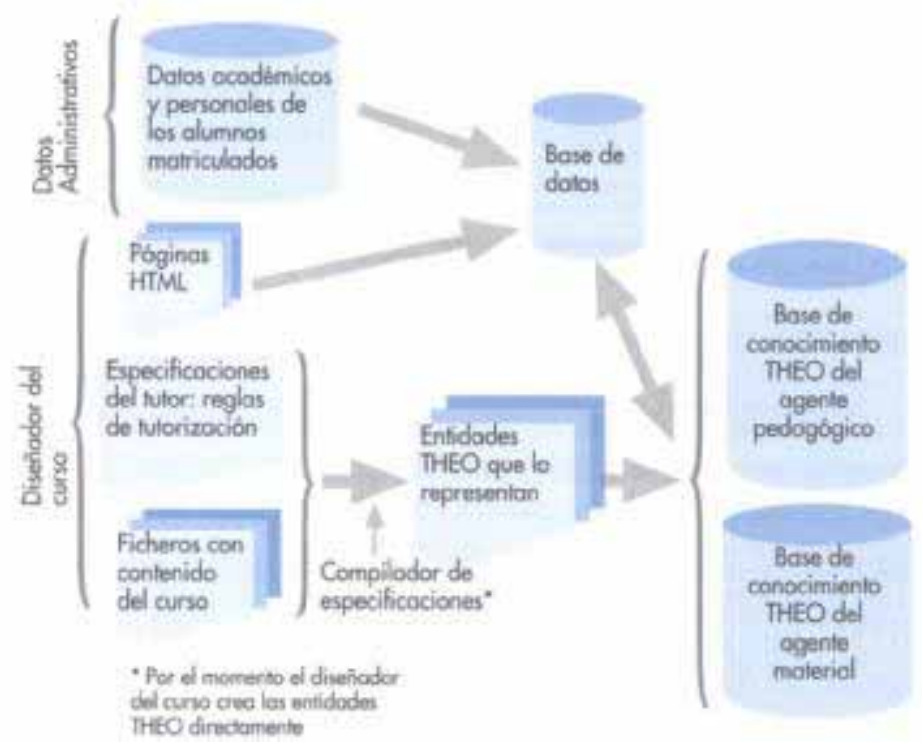

Figuro 3. Proceso de diseño de un curso con WebDL

A partir de ahí el alumno puede interaccionar con el sistema de la misma forma a como lo haría con un sitio web estático, mientras, WebDL va guardando las trazas de acceso del usuario. Dichas trazas serán utilizadas posteriormente como ejemplos de entrenamiento a la hora de realizar las tareas de aprendizaje.

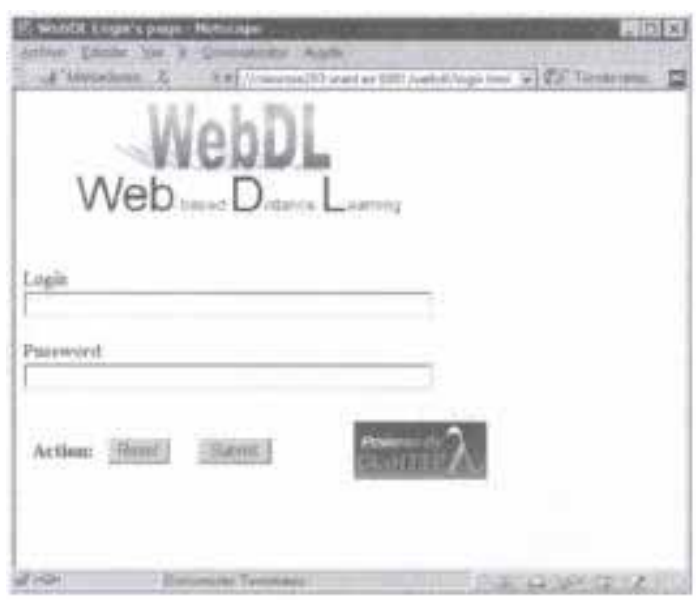

Figuro 4. Pantalla de entrada a WebDL

Cuando el alumno solicita una de las páginas del curso, el agente coordinador reenvía la petición a los agentes que pueden dar una respuesta al alumno. En el caso de la tarea de soporte adaptativo a la navegación, sobre los contenidos del curso, el agente encargado de decidir cuál es el siguiente enlace a aconsejar es el agente pedagógico.

La Figura 5 muestra la pantalla del sistema con un enlace recomendado. 


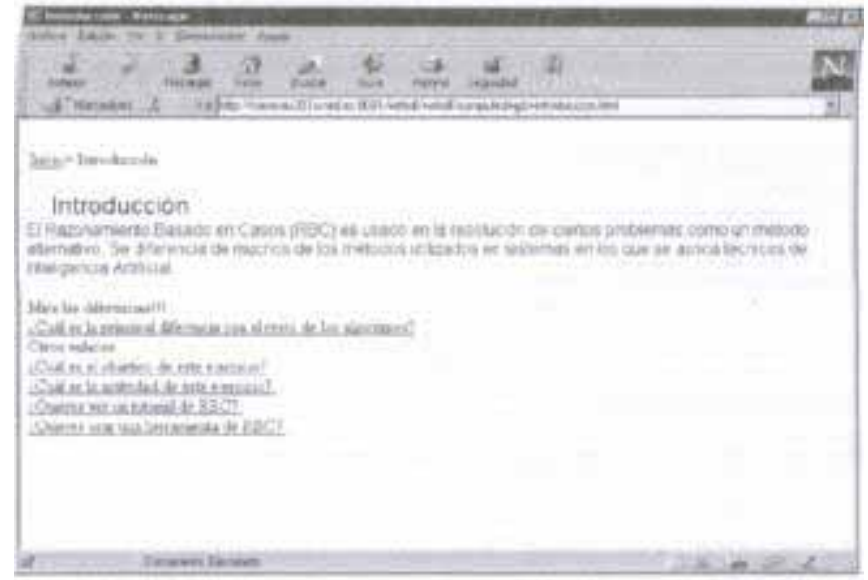

Figuro 5. Pantalla de WebDL con un enlace recomendado

La tarea de soporte adaptativo a la colaboración consiste en asistir al usuario del sitio web en su cooperación con el resto de usuarios. En WebDL distinguimos distintas clases de cooperación entre alumnos y profesores:

- Colaboración a través de anotaciones públicas en las prácticas.

- Colaboración mediante comunicaciones síncronas y asíncronas de las personas conectadas en un determinado momento (chats, foros, espacios de trabajo compartido,...).

- Colaboración mediante creación de grupos de trabajo dentro de los espacios de trabajo compartido en función de las similitudes entre los distintos alumnos.

El agente de contacto es el que responde al agente coordinador con las recomendaciones que pueden ayudar al alumno a comunicarse y a cooperar con otros alumnos o con los profesores.

Los alumnos en WebDL pueden realizar anotaciones en las páginas del curso siguiendo el enlace que se incluye en todas las páginas en que se permiten anotaciones. El profesor puede determinar qué páginas del curso pueden ser anotadas y cuáles no.

Cuando un alumno solicita una página que ha sido anotada anteriormente, el agente de contacto determina qué anotaciones pueden serle de interés, y le permite que realice él mismo una anotación (ver Figura 6).

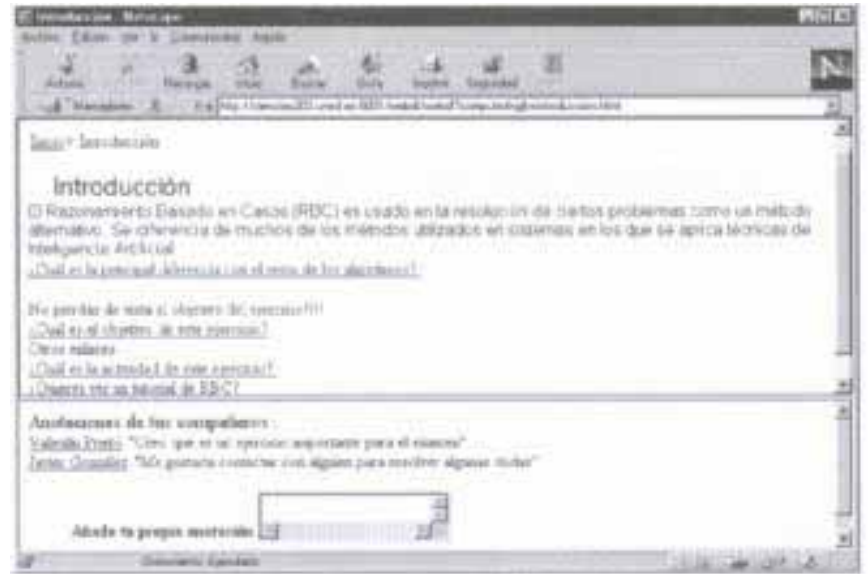


Figura 6. Pantalla de WebDL con una anotación recomendada

\section{CONCLUSIONES}

En este artículo se han descrito los fundamentos y las especificaciones de un servidor Web que contiene un sistema de educación adaptativo en la Web. La ventaja de este enfoque radica en distinguir tareas de adaptación no sólo para las tareas de asistencia en el estudio, sino también para cualquier otra actividad realizada en el sitio web, incluyendo el acceso a cualquier recurso disponible (páginas, repositorios, búsquedas, listas de distribución, etc.). El sistema se comporta como un sistema interactivo pensado para centrar la atención de la enseñanza en el rendimiento del alumno y para solventar los problemas que se están detectando en el uso de Internet para la enseñanza a distancia.

El diseño que se está utilizando combina el garantizar la usabilidad del sitio a través de un portal dinámico de acceso y una guía clara y explícita de la navegación realizada con la aplicación de un sistema adaptativo que proporciona un acceso personalizado a las necesidades de información y de comunicación de cada usuario en cada instante.

Se ha realizado un diseño de material y recursos telemáticos adaptados a las necesidades concretas de los usuarios potenciales del sistema: alumnos, profesores y profesores tutores de la UNED. Hasta el momento se están desarrollando los elementos relacionados con tres asignaturas de los estudios de informática de la UNED, cuya docencia depende del Departamento de Inteligencia Artificial.

\section{REFERENCIAS BIBLIOGRÁFICAS}

BOTICARIO, J.G. (1997a). Internet y la universidad a distancia. A Distancia, pags 64—69.

BOTICARIO, J.G. (1997b). Material didactico y servicios para la educacion a distancia en Internet. A Distancia, pags $70-76$.

BOTICARIO, J.G. y GAUDIOSO, E. (1999). Towards personalized distance learning on the web. En Mira, J. y Sanchez-Andres, J., (eds.), Foundations and Tools for Neural Modeling, number 1607 en Lecture Notes in Computer Science, pags 740—749. Springer Verlag.

BOTICARIO, J.G. y GAUDIOSO, E. (2000a). Adaptive web-site for distance learning. Campus-Wide Information Systems. Aceptado y pendiente de publicación durante este año.

BOTICARIO, J.G. y GAUDIOSO, E. (2000b). A multiagent architecture for a web-based adaptive educaticon system. En Rogers, S. and Iba, W., (eds.), Adaptive User Interfaces, Papers from the 2000 AAAI Spring Symposium, TR SS-00-01, pags 24-27. AAAI Press.

BOTICARIO, J.G. y GAUDIOSO, E. (2000c). Towards a personalized web-based educational system. En Cairo O., Sucar L.E.y Cantu F.J. (eds.), MICAI00: Advances in Artificial intelligence, number 1793 en Lecture Notes in Artificial Intelligence, pags 729—740. Springer Verlag, 2000.

BOTICARIO, J.G., GAUDIOSO, E. Y HERNÁNDEZ F. (2000). Adaptive Navigation Support and Adaptive Collaboration Support in WebDL. En Proceedings of the International Con ference on Adaptive Hypermedia and Adaptive Web-based Systems, Lecture Notes in Computer Science (LNCS), Trento, Italy. Springer Verlag; 28-30 Agosto 2000. Aceptado y pendiente de publicación para este año.

BRUSILOVSKY, P. (1996). Methods and techniques of adaptive hypermedia. User Modeling and UserAdapted Interaction, pags 87-129. Kluwer academic publishers.

BRUSILOVSKY, P. (1998). Adaptive educational systems on the world-wide-web: A review of available technologies. En Proceedings of Workshop WT.A/W-Based Tutoring at Fourth International Con ference on ITS (ITS’98), San Antonio, TX. MIT Press. 
BRUSILOVSKY, P., SCHWARZ, E., AND WEBER, G. (1996). A tool for developing adaptive electronic textbooks on www. En Proceedings of WebNet96, pags 64-69, San Francisco, CA. World Conference of the Web Society.

CHEN, W. y MIzoGucHI, R. (1999). Communication ontology for learner model agent in multiagent architecture. En Proceedings of the International Con ference on Artificial Intelligence in Education (AIED99), Le Mans, France.

DENT, L., BOTICARIO, J.G., MCDERMOTr, J., MITCHELL, T. M., y ZABowsKI, D. T. (1992), A personal learning apprentice. En Proceedings of the Tenth National Conference on Artificial Intelligence, pags 96-103, San Jose, CA. MIT Press.

FININ, T., FRITZSON, R., MCKAY, D., y McENTIRE, R. (1994). KqmI as an agent communication language. En Proceedings of the Third International Con ference on Information and Knowledge Management, pags 64-69. ACM Press.

GENESERETH, M. y FIKES, R. (1992). Knowledge interchange format, version 3.0 reference manual. Technical Report KSL-92-86, Knowledge Systems Laboratoly.

GIRALDEZ, 3.1. y Boruo, D. (1998). Distributed reinforcement learning in multi agent decision systems. En Coelho, H., editor, Progress in Artificial Intelligence, number 1484 in Lecture Notes in Computer Science. Springer

Verlag.

MCARTHUR, D., LEWIS, M., y BIsHAY, M. (1993). The roles of artificial intelligence in education: current progress and future prospects. Technical Report DRU-472-NSF, RAND, Santa Monica, CA.

MITCHElL, T.M., ALlEN, J., CHALASANI, P., CHENG, J., ETzIONI, O., RINGUEHE, M., y SCHLIMMER, J.C. (1990). Theo: A framework for self-improving systems. En VanLehn, K., editor, Architectures for Intelligence. Erlbaum, Hillsdale, NJ.

NAKABAYASHI, K. (1996). An intelligent tutoring system on the www supporting ubteractive simulation environments with a multimedia viewer control mechanism. En Proceedings of WebNet96, página 366, San Francisco, CA. World Conference of the Web Society.

SCHANK, R.C. Y CLEARY, C. (1995). Engines for education. Lawrence Erlbaum Associates, Milisdale, New Jersey.

WEBER, G. y SPECHT, M. (1997). User modeling and adaptive navigation support in www-based tutoring systems. En Proceedings of the Sixth International Con ference on User Modeling, pags 289300 ,

Chia

Laguna,

Sardinia,

Italy.

\section{PERFIL ACADÉMICO Y PROFESIONAI. DE LOS AUTORES}

Jesús González Boticario Profesor Titular de Escuela Universitaria dentro del Departamento de Inteligencia Artificial de la Universidad Nacional de Educación a Distancia. Es director de la Unidad Técnica de Investigación y Formación en Recursos Tecnológicos del Instituto Universitario de Educación a Distancia. Sus principales líneas de investigación son el aprendizaje automático, el desarrollo de arquitecturas multiagente y Sistemas interactivos de Enseñanza/aprendizaje.

\section{Jesús González Boticario}

Departamento de Inteligencia Artificial

Facultad de Ciencias, UNED

C/Senda del Rey 9. 28040 Madrid. España

Teléfono: 34-1-3987197

Fax: 34-1- 3986697 
Correo electrónico: jgb@dia.uned.es http://www.ia.serv.dia.uned.es/jgb/

Elena Gaudioso Vázquez Becaria predoctoral de la Universidad Nacional de Educación a Distancia. Sus principales líneas de investigación son el desarrollo de sistemas aprendices para el apoyo a la educación a distancia a través de Internet, la aplicación de sistemas multiagente y de técnicas de aprendizaje automático para el desarrollo de sistemas de educación.

Elena Gaudioso Vázquez

Departamento de Inteligencia Artificial

Facultad de Ciencias, UNED

C/Senda del Rey 9. 28040 Madrid. España

Teléfono: 34-1-3987242

Fax: 34-1- 3986697

Correo electrónico: elena@dia.uned.es

Félix Hernández del Olmo Becario predoctoral de la Universidad Nacional de Educación a Distancia. Sus principales lineas de investigación son el aprendizaje automático, los sistemas multiagente con aprendizaje y su aplicación al desarrollo de sistemas interactivos de educación.

Félix Hernández del Olmo

Departamento de Inteligencia Artificial

Facultad de Ciencias, UNED

C/Senda del Rey 9. 28040 Madrid. España

Teléfono: 34-1-3988345

Fax: 34-1- 3986697

Correo electrónico:felixh@dia.uned.es 